\title{
Determining the Effect of Ashwagandha Plant Powder on Men with Semen Disorders
}

\author{
Research Article
}

\section{Mehdi Rashidi', Majid Emtiazy2,3*, Mahmood Khodadoost ${ }^{4}$, Hossien Fallahzadeh 5 , Ali Mohammad Mirjalili6}

1. Ph.D. Student of Persian Medicine, Department of Persian Medicine, School of Persian Medicine, Shahid Sadoughi University of Medical Sciences, Ardakan, Yazd, Iran.

2. The Research Center of Persian Medicine, Shahid Sadoughi University of Medical Sciences, Yazd, Iran.

3. Associate professor, Department of Persian Medicine, School of Persian Medicine,

Shahid Sadoughi University of Medical Sciences, Ardakan, Yazd, Iran.

4. Associate Professor of Traditional Medicine, School of Traditional Medicine, Traditional Medicine \& Materia

Medical Research Center, Shahid Beheshti University of Medical Sciences, Tehran, Iran.

5. Professor of Biostatistics, Department of Biostatistics and Epidemiology,

School of Health Shahid Sadoughi University of Medical Sciences, Yazd, Iran.

6. Research and Clinical Center for Infertility, Shahid Sadoughi University of Medical Sciences, Yazd, Iran.

\begin{abstract}
Introduction: Infertility, which is defined as the inability to conceive after one year of intercourse without the use of contraceptive methods, affects $15 \%$ of couples. $30-40 \%$ of the causes of male infertility are related to sperm disorders. The most common cause of infertility in men is their inability to produce enough healthy, active and active sperm, so the aim of this study was to determine the effect of ashwagandha powder on men with semen disorders. Materials and Methods: This study is a double-blind, clinical, randomized trial. Subjects were entered the study after obtaining informed consent and having inclusion and exclusion criteria. All subjects were clinically examined by a urologist. Blood and urine tests were also performed to ensure the health status of the individuals. Subjects did not take any PDE-V inhibitors or glucocorticoids, chemical, herbal and hormonal drugs for infertility treatment in the 4 weeks before the start of the study and until the end of the study. Finally, demographic information was obtained from all individuals and statistical calculations were performed using the statistical software SPSS. Results: In general, our results showed that the consumption of ashwagandha powder in the first experimental group was $41.3 \pm$ $16.66 \%$ in the pre-test stage, $46.53 \pm 13.42 \%$ in the post-test stage and $45.5 \% \pm 11.99 \%$ in the follow-up stage. These values were $41.23 \pm 19.85 \%$ in the placebo group of the pre-test stage, $41.0 \% \pm 19.15$ in the post-test stage and $40.37 \pm 19.41 \%$ in the follow-up stage. This indicates that ashwagandha increased semen performance scores and sperm motility in different stages of the test compared to the second experimental group. Conclusion: The use of medicinal plants with male fertility enhancement properties can be used as a substitute or supplement for chemical drugs that affect male fertility. On the other hand, it is recommended that chemical drugs with fertility-reducing properties be less used or not used in men with infertility disorders.
\end{abstract}

Key Words: Ashwagandha plant, Men, Semen.

\section{Introduction}

Today, advances in science and technology, along with declining mortality and rapid population growth, have led to research on fertility and infertility through hormonal, chemical and immunological approaches. Currently, most of these methods are used by women. In addition to the fact that many of these

* Corresponding Author:

\section{Majid Emtiazy}

The Research Center of Persian Medicine,

Shahid Sadoughi University of Medical Sciences,

Yazd, Iran.

Email Id:dr.emtiazi@yahoo.com methods are useless, but in some cases they are harmful. Therefore, the World Health Organization (WHO) has considered a population control program based on traditional medical studies and the use of plant properties (1-3). Infertility, defined as the inability to conceive after one year of intercourse without the use of contraception, affecting $15 \%$ of couples (4). Furthermore, $30-50 \%$ of the causes of infertility are related to male infertility and also $30-40 \%$ of the causes of male infertility are related to sperm disorders (5). The most common cause of infertility in men is their inability to produce enough healthy, active, and motile sperm (6). The ability to conceive in men depends in part on the number, quality, motility and shape of the sperm, and disruption of any of these factors can lead to male infertility (7). 
Lack of testicular development and growth, diseases of the reproductive system, increased scrotal temperature, immune problems, endocrine disorders, lifestyle, environmental factors and nutrition are considered as the main causes of male infertility that negatively affect sperm parameters $(8-10)$. There are many medicinal plants in the world with anti-fertility and fertility enhancing properties. These plants have historically been used to reduce and increase fertility in men, so that modern scientific research has been tested to confirm the anti-fertility and fertility enhancing effects of some of these plants (11-12). Many people now use herbs or their derivatives to increase or decrease fertility as well as libido (13). Various medicinal plant extracts with anti-fertility and fertility enhancing effects have been studied in both males and females.

Some of these plants have spermicidal properties, others increase motility and change the number of sperm. Some plants also alter testicular hormones (14). It is necessary to further study the use of biologically active plant materials in the field of male fertility and to identify natural plant materials with estrogenic and anti-estrogenic properties (8). The WHO reports that despite the increasing use of herbal medicines, there is still a significant lack of research information, and the role of articles examining herbal medicines is crucial. Due to the clear negative effects of chemical drugs on humans, the tendency to use herbal medicines is increasing among women and men. Herbal medicines are a good alternative to regular medicines. Combination therapy of both herbal and conventional medicine has made herbal medicine more important in today's world. Due to men's fear of infertility (15), it is very important to pay attention to medicinal plants that are effective in male fertility. Therefore, the present study aimed to determine the effect of ashwagandha plant powder on men with semen disorders.

\section{Materials and Methods}

This randomized, and double-blind clinical trial was performed on men with semen disorders (number or motility) who were referred to the Yazd Infertility Center, Iran for one month in the fall of 2018.

A questionnaire was used to collect data. The sampling was randomly performed and eligible individuals were included in the study according to inclusion and exclusion criteria.

\section{Inclusion criteria}

1- Age range 18 to 45 years, 2- The person is abnormal in at least one of the sperm parameters, including number or motility. Regarding the number of sperm in the semen, if it is less than 20 million per milliliter of sperm, the person is abnormal and if sperms have $60 \%$ motility, can be appropriate, 3 - The male is married for one year of unprotected sex with his wife and has not become pregnant, 4- A person's spouse is completely normal at the discretion of a gynecologist.

\section{Exclusion criteria}

1- Primary erectile dysfunction, 2- Congenital anomalies, 3- Uncontrolled diabetes mellitus, 4- Kidney failure, 5- Liver failure, 6- Cardiovascular diseases, 7Oligospermia due to obstructive reasons, 8- Allergy to ashwagandha plant, 9- Cerebrovascular injuries, 10Uncontrolled hypertension, and history of cryptorchidism, 11- Testicular and varicocele, 12People with a history of pelvic fractures, 13- Prostate surgeries, 14- History of prostate resection, 15- Penis reconstruction surgeries, 16- Genital infections, 17Having systemic diseases, 18- Request to leave the study, 19- History of chemotherapy, 20- Use of anticoagulants, 21- Unilateral testicular atrophy, 22Thyroid diseases, 23- Gallbladder diseases, 24- History of cancer, 25- Pelvic surgery, 26- Alcoholism, 27- Drug addiction, 28- Smoking

\section{Sample size}

The sample size was determined by considering the significance level of $5 \%$, test power of $80 \%$ and the $\mathrm{S}$ of 11. To achieve a significant difference, at least 10 units in the average number of sperm, 25 people in each group are required. With a $10 \%$ drop, 30 people in each group are examined. Formula used to determine sample size:

\section{Procedure}

After obtaining informed consent and having inclusion and exclusion criteria, patients entered the study. All people were clinically examined by a urologist to ensure their health. Blood and urine tests were also performed to ensure their health status. The subjects did not take any PDE-V inhibitors (Phosphodiesterase V) and glucocorticoids, chemical, herbal and hormonal drugs for infertility treatment in the 4 weeks before the start of the study and until the end of the study, which was in accordance with medical and ethical standards. This means that if someone was forced to use these drugs due to their own interests or desires, it was eventually excluded from the study, and this loss is taken into account in the design of the study. Demographic information was eventually obtained from all individuals.

The study was randomized into two groups, the first 30 people receiving placebo and the 30 people receiving ashwagandha. Randomization was performed using a computer-generated random number table, which divides individuals into two groups. People received ashwagandha five times a day, one capsule (one gram of concentrated ashwagandha powder) for 12 weeks.

The prescribed ashwagandha plant has a standardized state and the amount of alkaloids and its vitaminolides as well as the amount of short and long chain amino acids (threonine, valine, methionine, isoleucine, lysine, aspartic acid and arginine), iron, complex sugars (oligosaccharides, oligosaccharides), vitamin $\mathrm{A}$ and calcium will be adequate and equal in standard.

In the placebo group, 5 grams of the placebo was given five times a day (one gram) for 12 weeks. 
During the study period, people had four visits, the first visit was before starting the powder, the second visit was four weeks after the first use, the third visit was eight weeks after the first use and the fourth visit was twelve weeks after the first use.

At each visit, individuals underwent sperm analysis and physical examination. It should be noted that the pattern of sperm collection is also done according to WHO guidelines.

\section{Data analysis}

Kolmogorov-Smirnov test was used to check the normality of the data. Intention-to-treat analysis was used for sample volume loss. Data analysis was performed using mixed-design analysis of variance and statistical calculations were performed using SPSS statistical software.

\section{Ethical considerations}

Informed consent of patients was obtained. This study started after obtaining the code of ethics from the University of Medical Sciences and the IRCT code. The principles of Helsinki have been observed.

\section{Results}

A total of 60 people entered the study and were divided into two groups. Mean and standard deviation of sperm function questionnaire scores were examined in two experimental groups in pre-test, post-test and follow-up stages (Table 1 and Figure 1). The results show that the mean scores of sperm performance in the post-test and follow-up stages increased compared to the pre-test stage in the first experimental group (herbal powder consumption) but in the second experimental group (placebo) the mean sperm function scores did not change much. It is noteworthy that the increase in score on the sperm function scale indicates normal sexual function.

Table 1: Descriptive indices of sperm function scores in the two groups in the pre-test, post-test and follow-up stages

\begin{tabular}{|c|c|c|c|c|c|c|c|}
\hline & & \multicolumn{2}{|c|}{ Pre-test } & \multicolumn{2}{c|}{ Post-test } & \multicolumn{2}{c|}{ Follow-up } \\
\hline Group & Number & Mean & SD & Mean & SD & Mean & SD \\
\hline 1 & 30 & 41.3 & 16.66 & 46.53 & 13.42 & 45.5 & 11.99 \\
\hline 2 & 30 & 41.23 & 19.85 & 41.0 & 19.15 & 40.37 & 19.41 \\
\hline
\end{tabular}

Figure 1: Mean scores of sperm function in the two groups in the pre-test, post-test and follow up stages

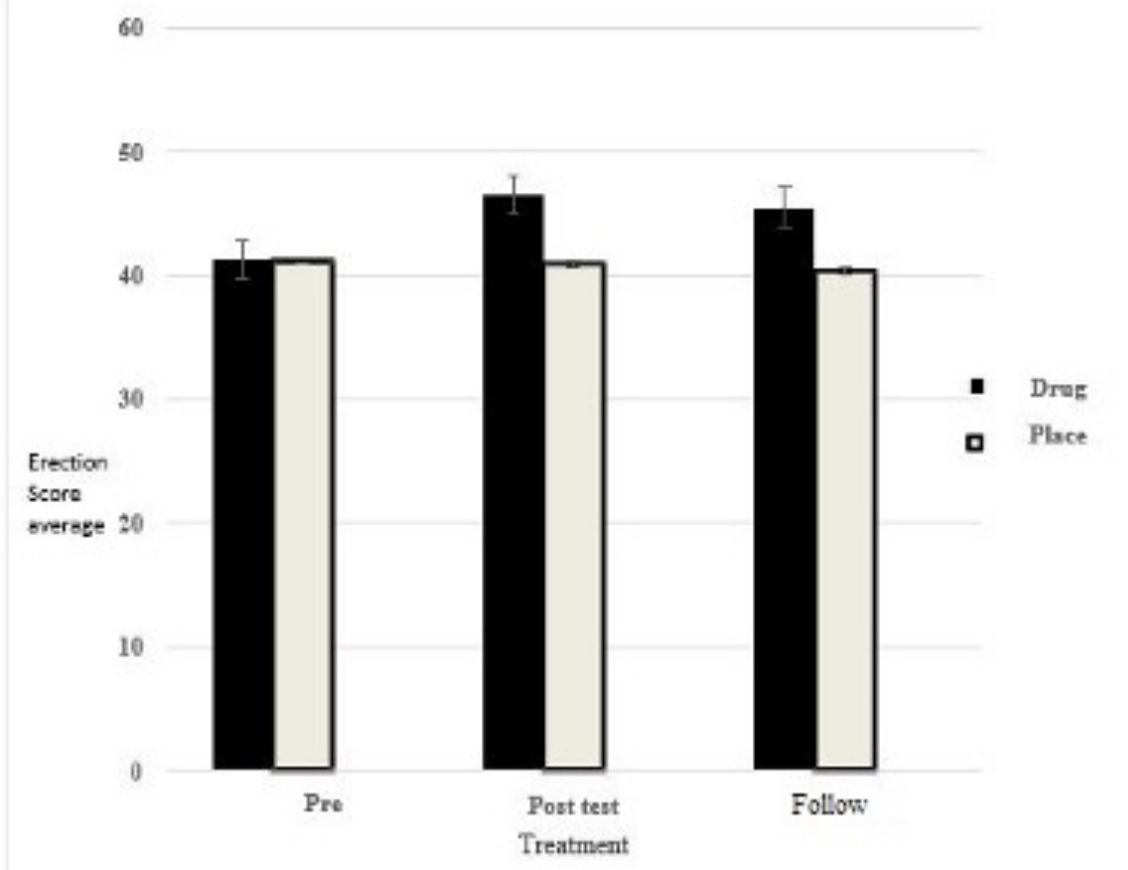

In order to investigate the significant difference between the mean scores of sperm function and to evaluate semen abnormality in the first and second experimental groups in the pre-test, post-test and follow-up stages, the mixed analysis of variance method was used. The three stages of pre-test, post-test and follow-up were considered as inter-subject factors and grouping of subjects in three groups was also considered as an inter-subject factor. First, the assumptions of homogeneity of variance and sphericity were examined by Mauchly's sphericity test for intragroup factor. The results of Table 2 show that $\mathrm{F}$ scores were not significant for pre-test scores but were significant for post-test and follow-up scores. Therefore, the assumption of homogeneity of variances was true only for pre-test scores. Regarding the equal volume of groups, this assumption can also be true for post-test and follow-up scores. 
Mehdi Rashidi et.al., Determining the Effect of Ashwagandha Plant Powder on Men with Semen Disorders

Table 2: Results of the same assumption of the variance matrix

\begin{tabular}{|l|c|c|c|c|}
\hline \multicolumn{1}{|c|}{ Variable } & F & df1 & df2 & sig \\
\hline Pre-test scores & 2.39 & 2 & 87 & 0.098 \\
\hline Post-test scores & 4.8 & 2 & 87 & 0.011 \\
\hline Follow up scores & 7.507 & 2 & 87 & 0.001 \\
\hline
\end{tabular}

Table 3 shows that the sphericity hypothesis is not true $\left(\chi^{2}(2)=16.677, \mathrm{P}<0.05\right)$ so instead of a degree of freedom, an index called Greenhouse-Geisser correction should be used and the results of mixed analysis of variance should be calculated due to the lack of sphericity assumption.

Table 3: Results of the Mauchly's sphericity for testing the sphericality hypothesis

\begin{tabular}{|c|c|c|c|c|c|}
$\begin{array}{c}\text { Intra-group } \\
\text { agent }\end{array}$ & Mauchly' test & Chi-square & df & Sig & Greenhouse-Geisser \\
\hline Stages & 0.824 & 16.677 & 2 & 0.001 & 0.85 \\
\hline
\end{tabular}

$\mathrm{F}$ at the level of 0.05 is significant in relation to the internal factor calculated for the effect of steps (pre-test, post-test and follow-up) $(\mathrm{F}=3.601, \mathrm{P}<0.05$; Table 4). As a result, there was a significant difference in the performance of semen disorders (number or mobility) in the three stages of treatment between the mean scores of pretest, post-test and follow-up. A summary of the results of the mixed analysis of variance for intragroup and intergroup factors is presented in Table 4. The Bonferroni test was used to examine the differences between the means (Table 5). The results of Table 5 show a significant difference at the level of 0.05 between the performance scores of semen disorder (number or mobility) between the pre-test and post-test stages. But there was no significant difference between pre-test scores and follow-up and post-test scores with follow-up.

Table 4: Summary of the results of mixed analysis of variance with intragroup and intergroup factors

\begin{tabular}{|c|c|c|c|c|c|c|}
\hline Factors & Change Sources & Total squares & $\begin{array}{c}\text { Degrees of } \\
\text { freedom }\end{array}$ & $\begin{array}{l}\text { Average } \\
\text { squares }\end{array}$ & $\mathbf{F}$ & meaningful \\
\hline Intragroup factors & $\begin{array}{l}\text { Treatment steps } \\
\text { Interaction of stages } \\
* \text { Group }\end{array}$ & $\begin{array}{c}137.6 \\
337.111\end{array}$ & $\begin{array}{c}1.7 \\
3.401\end{array}$ & $\begin{array}{l}80.928 \\
99.134\end{array}$ & $\begin{array}{l}3.601 \\
4.411\end{array}$ & $\begin{array}{l}0.037 \\
0.004\end{array}$ \\
\hline Intergroup factors & $\begin{array}{c}\text { Group } \\
\text { Error }\end{array}$ & $\begin{array}{c}576.622 \\
7786.511\end{array}$ & $\begin{array}{c}2 \\
87\end{array}$ & $\begin{array}{c}288.311 \\
894.96\end{array}$ & 0.322 & 0.725 \\
\hline
\end{tabular}

Comparison of the adjusted means shows that the function scores of semen disorder (number or mobility) in the pre-test stage $(\mathrm{M}=41.68)$ are lower than the post-test stage $(\mathrm{M}=43.42)$. But there was no significant difference between the function scores of semen disorder (number or mobility) in the pre-test and follow-up and post-test and follow-up stages (Table 5). Also, in relation to the interaction of stage factors and group, the value (F) calculated for the effect of stages (pre-test, post-test and follow-up) between the experimental and control groups was found to be significant at the level of $0.05(\mathrm{~F}=4.411, \mathrm{P}<0.05)$. As a result, a significant difference was observed between the mean scores of pre-test, post-test and follow-up scores of semen dysfunction in the two groups. For the intergroup factor, the value of $\mathrm{F}$ calculated at the level of 0.05 was not significant. As a result, there is no significant difference between the overall mean of semen dysfunction (number or mobility) in the experimental and control groups, so that the adjusted means of total semen dysfunction performance scores (number or motility) in the two experimental and control group was not much different from each other.

Modified means of semen dysfunction performance scores (number or mobility) in the experimental and control groups at different stages are shown in Figure 2. Semen dysfunction scores (number or motility) increased in the first experimental group (herbal powder consumption) in the post-test and follow-up stages compared to the other group (placebo experimental group) (Figure 2).

Table 5: Summary of the Bonferroni test results

\begin{tabular}{|c|c|c|c|}
\hline Stages & Mean difference & Standard error & Sig \\
\hline Pre and post text & $*-1.733$ & 0.667 & 0.033 \\
\hline Pre and follow up test & -0.067 & 0.753 & 0.481 \\
\hline Post and follow up test & $0.667 \quad *=\mathrm{P}>0.05$ & 0.511 & 0.586 \\
\hline
\end{tabular}


Figure 2: Modified means of erection performance scores in experimental and control groups in pre-test, posttest and follow-up stages.

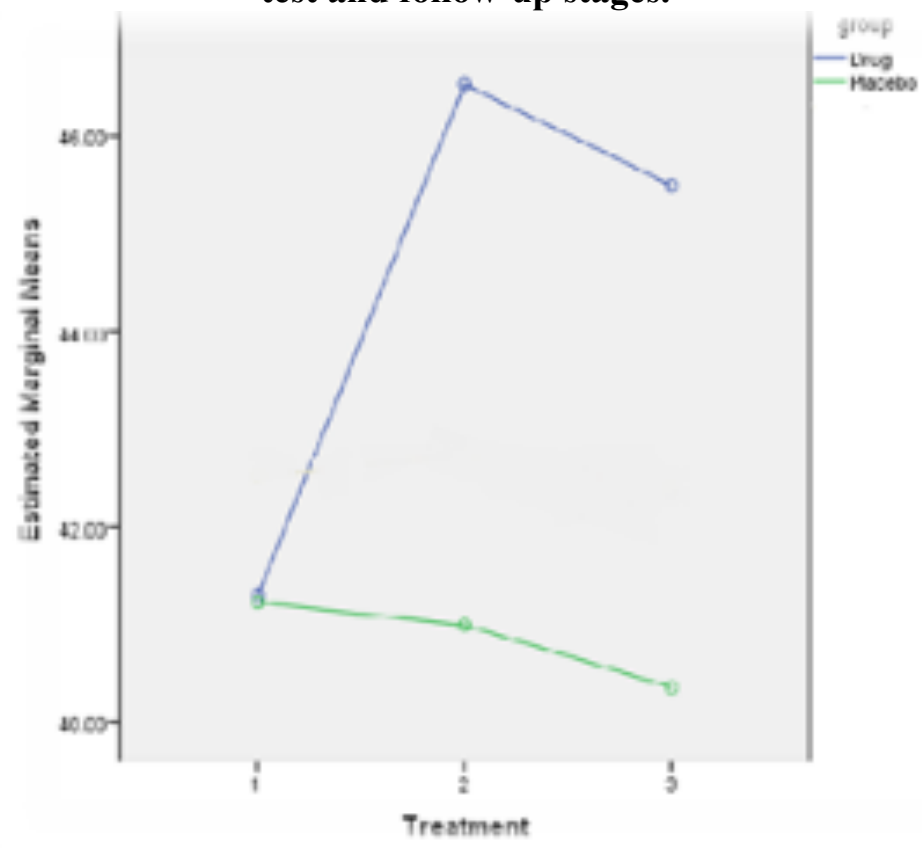

\section{Discussion}

Regarding the support of the World Health Organization for maintaining public health and reproductive health, today the use of medicinal plants is considered as a substitute or supplement for synthetic drugs that affect fertility. Various studies have shown that some medicinal plants with fertility enhancing properties in men prevent the formation of free radicals and lipid peroxidation and reduce oxidative stress by improving antioxidant activity leading to prevention of sperm cell damage.

With the use of medicinal plants effective in male fertility, parameters such as sperm survival and mortality, pituitary hormone levels, histological changes in the testes and sperm depletion are affected. Therefore, these herbs can help improve sperm parameters in infertile men $(16,17)$. Therefore, the aim of this study was to determine the effect of ashwagandha powder on men with semen disorders.

Our results showed that the consumption of ashwagandha plant powder in the first experimental group was $1.41 .3 \pm 16.66 \%$ in the pre-test stage, 46.53 $\pm 13.42 \%$ in the post-test stage and $45.5 \pm 11.99 \%$ in the follow-up stage.

In the second group (placebo user), this rate was $41.23 \pm 19.85 \%$ in the pre-test stage, $41.0 \pm 19.15$ $\%$ in the post-test stage and $40.37 \pm 19.41 \%$ in the followup stage. This indicates that of ashwagandha increased semen performance scores and sperm motility in different stages of the test compared to the second experimental group. Various studies have been performed in this regard, including the study of Ambiye et al. In 2013 in India, which showed that the administration of ashwagandha root extract at a dose of $675 \mathrm{mg}$ three times a day for 11 days in infertile men with oligospermia increases the mean semen volume by
$53 \%$, sperm count by $167 \%$ and sperm motility by $57 \%$ (18).

The results of this study were consistent with the results of the current study, except for the increase in semen volume.

In a study in India, the effect of ashwagandha root consumption in men was investigated, where the amount of $5 \mathrm{~g}$ daily consumption of ashwagandha root powder for 3 months increased sperm concentration $(17 \%)$ and sperm motility $(9 \%)$ in men with normal sperm under the influence of various environmental stresses. Also, this plant has increased the sperm concentration (36\%) and sperm motility $(13 \%)$ in infertile men without stress. In men affected by psychological stress, a $20 \%$ increase in concentration and a $10 \%$ increase in sperm motility in infertile men with smoking history. The results of our study confirmed the results of mentioned study, although in our study the stress variable was not considered (19).

In the present study, in order to investigate the significant difference between the mean scores of sperm function in patients and semen disorders in the first and second groups in the experimental stages, the method of mixed-design analysis of variance was used.

The three stages of the test were considered as an intra-subject factor in the male and the grouping of the subjects in the three groups was considered as an inter-subject factor. Assumptions of variance and sphericity homogeneity were tested by Mauchly's sphericity test for intragroup factor. The results showed that $\mathrm{F}$ scores are not significant only for pre-test scores and are significant for post-test and follow-up scores. Therefore, the assumption of homogeneity of variance is true only for pre-test scores. Considering the equal volume of groups, this assumption can also be considered true for post-test and follow-up scores. The findings of this study confirmed that ashwagandha 
plant powder significantly increased semen performance and sperm motility.

The results of a 2010 clinical trial by Ahmad et al. in India on infertile men with normospermia, oligospermia or asthenospermia showed that 3-month use of ashwagandha root powder increased semen volume in normospermic and oligospermic men and also increased sperm concentration and motility in infertile oligospermic and asthenospermic men compared to before intervention. The results of this study were consistent with our current study except for an increase in semen volume (20).

Overall, the results of various studies show that this plant with its ability to remove obstructions in the body's ducts, which is probably its anti-inflammatory property, can help in the treatment of infertility $(21,22)$. Furthermore, in infertile men, the activity of alanine aminotransferase, lactate dehydrogenase and isocitrate dehydrogenase are significantly reduced compared to the group of fertile men. Decreased levels of these enzymes are considered to be another factor involved in male infertility. Alanine protects sperm from damage caused by oxidative stress and ultimately increases sperm count and motility (23).

The nutrients of ashwagandha such as multiple amino acids, fatty acids, minerals and vitamins can have positive effects on improving semen and the male reproductive system $(24,25)$.

\section{Conclusion}

The use of medicinal plants with the property of enhancing male fertility can be used as a substitute or supplement to chemical drugs that affect male fertility. On the other hand, it is recommended that chemical drugs with fertility-reducing properties be less used or not used in men with infertility disorders.

\section{References}

1. Stephan A. Prospects for pharmacological male contraception. Drugs. 1994; 48(6): 851-63.

2. $\mathrm{Wu}$ FC. Male contraception: current status and future prospect. Clin Endocrinol. 1988; 29(4): 443-65.

3. Sathlyaraj K, Sivaraj A, Madhumitha G, Vinothkmar P, Mary Sral A, Devi K, et al. Antifertlity effect of aqueous leaf extract of Aegle Marmelos on male albino rats. International Journal of Current Pharmaceutical Research. 2010; 2(1):26-9.

4. Kim SJ, Kim MR, Hwang SY, Bae WJ, Kim S, Hong SH, et al. Preliminary report on the safety of a new herbal formula and its effect on sperm quality. World J Mens Health. 2013; 31(3): 254-61.

5. Godmann M, Lambrot R, Kimmins S. The dynamic epigenetic program in male germ cells: Its role in spermatogenesis, testis cancer, and its response to the environment. Microsc Res Tech. 2009; 72(8): 603-19.

6. Bastampoor F, Sadeghi H, Hosseini SE. The petroselinum crispum L. hydroalcoholic extract effects on pituitary-gonad axis in adult rats. Armaghane Danesh. 2014; 19(4): 305-13.

7. Oyeyemi MO, Olukole S, Esan O. Sperm morphological studies of west African Dwarf Bucks treated with pumpkin plant (Cucurbita pepo). Int $\mathrm{J}$ Morphol. 2008; 26(1):121-6.

8. Marah I, Marbeen A, Mossa M, Marbut I, Allahwerdy Y. The probable therapeutic effects of date palm pollen in the treatment of male infertility. Tikrit J Pharmaceutical Sci. 2005; 5(1):30-5.

9. Sharpe RM, Franks S. Environment, lifestyle and infertility--an inter-generational issue. Nat Cell Biol. 2002; 4: 33-40.

10. Low BS, Das PK, Chan KL. Standardized quassinoid-rich Eurycoma longifolia extract improved spermatogenesis and fertility in male rats via the hypothalamic-pituitary-gonadal axis. J Ethnopharmacol. 2013; 145(3): 706-14.

11. Jain S. Medicinal plants with potential anti-fertility activity: a review. Int J Green Pharmacy. 2015; 9(4): 223-8.

12. Modaresi M, Messripour M, Asadi Marghmaleki M, Hamedanian MK. Effect of saffron (Crocus Sativus) extract on level of FSH, LH and testosterone in mice. Sci J Zanjan Univ Med Sci. 2008; 16(63); 11-18.

13. Kachroo M, Agrawal SS. Anti-implantation activity of different extract of the peels of citrus medica, Linn. Int J Pharm Tech Res. 2011; 3(1): 535-9.

14. Khaki A, Fathiazad F, Nouri M, Ozanci CC, Ghafari-Novin M, et al. The effects of Ginger on spermatogenesis and sperm parameters of rat. Iran J Reprod Med. 2009; 7(1): 7-12.

15. Hosseini H, Abdi F. Experiences of vasectomy: a phenomenological study. N Am J Med Sci. 2012; 4(12): 619-23.

16. Chauhan NS, Rao CV, Dixit VK. Effect of Curculigo orchioides rhizomes on sexual behaviour of male rats. Fitoterapia. 2007; 78(7-8): 530-4.

17. Parandin R, Ghorbani R, Sadeghipour Roodsari HR. Effects of alcoholic extract of Achillea Millefolium flowers on fertility parameters in male rats. SSUJ. 2011; 19(1): 84-93.

18. Ambiye VR, Langade D, Dongre S, Aptikar P, Kulkarni M, Dongre A. Clinical evaluation of the spermatogenic activity of the root extract of Ashwagandha (Withania somnifera) in oligospermic males: a pilot study. Evidence-Based Complementary and Alternative Medicine. 2013.

19. Mahdi AA, Shukla KK, Ahmad MK, Rajender S, Shankhwar SN, Singh V, et al. Withania somnifera improves semen quality in stress-related male fertility.Evidence-Based Complementary and Alternative Medicine. 2011.

20. Ahmad MK, Mahdi AA, Shukla KK, Islam N, Rajender S, Madhukar D, et al. Withania somnifera improves semen quality by regulating reproductive hormone levels and oxidative stress in seminal plasma of infertile males. Fertility and sterility. 2010; 94(3): 989-96. 
21. Khare C. Indian Medicinal Plants-An Illustrated Dictionary. 1st Indian Reprint Springer (India) Pvt. Ltd, New Delhi, India. 2007;28.

22. Narinderpal K, Junaid N, Raman B. A Review on pharmacological profile of Withania somnifera (Ashwagandha). Res Reviews J Bot Sci. 2013; 2: 6-14.

23. Gupta A, Mahdi AA, Shukla KK, Ahmad MK, Bansal N, Sankhwar P, et al. Efficacy of Withania somnifera on seminal plasma metabolites of infertile males: a proton NMR study at $800 \mathrm{MHz}$. Journal of ethnopharmacology. 2013; 149(1): 208-14.

24. Imtiyaz S, Ali SJ, Aslam M, Tariq M, Chaudhary SS. Withania somnifera: a potent unani aphrodisiac drug. Int Res J Pharm App Sci. 2013; 3(4):59-63.

25. Mishra L-C, Singh BB, Dagenais S. Scientific basis for the therapeutic use of Withania somnifera (ashwagandha): a review. Alternative medicine review. 2000; 5(4): 334-46. 